\title{
The Utility of Prostate-Specific Antigen Screening and Prostate Cancer Treatment in Elderly Patients
}

\author{
Annelies Vellekoop • Stacy Loeb
}

Published online: 9 April 2013

(C) Springer Science+Business Media New York (outside the USA) 2013

\begin{abstract}
Screening and treatment of prostate cancer remain controversial, particularly for elderly men. Since the previous United States Preventive Services Task Force recommendation in 2008 against prostate-specific antigen (PSA) screening after age 75 years, there has not been a substantial reduction in the frequency of PSA testing in this age group. A substantial proportion of elderly men are overdiagnosed and overtreated. On the other hand, men aged $>75$ years have more aggressive disease. Although screening has questionable benefit for elderly men with significant comorbidities, healthy men $>75$ years may benefit from definitive therapy. This review discusses prostate cancer screening and management decisions for elderly men, including the use of nomograms and predictive tools for life expectancy.
\end{abstract}

Keywords Prostate cancer · Screening · Elderly · Comorbidity $\cdot$ Treatment $\cdot$ Survival

\section{Introduction}

Prostate cancer $(\mathrm{PCa})$ is highly prevalent in the aging population in the United States. It is the second most commonly diagnosed malignancy and is the second most common cause of cancer death in U.S. men, with 238,590 new cases and 29,720 deaths estimated for 2013 [1]. For many years it has been a challenge to identify the optimal treatment for men with PCa. This relates to the heterogeneity of the

A. Vellekoop $\cdot$ S. Loeb

Department of Urology, New York University (AV, SL) and Manhattan

Veterans Affairs Medical Center (SL), New York, NY, USA

S. Loeb $(\bowtie)$

550 1st Ave (VZ30, 6th floor 612),

New York, NY 10016, USA

e-mail: stacyloeb@gmail.com disease ranging from slow growing, indolent tumors to highly aggressive and potentially fatal cases. At one end of the spectrum, patients with low-grade, low volume tumors may be cured with treatment, but may also be safely managed without treatment. Patients with aggressive but still localized tumors may benefit most from definitive treatment, such as radical prostatectomy (RP), brachytherapy (BT), or external beam radiation therapy (EBRT) potentially combined with androgen deprivation therapy (ADT). However, some patients present with metastatic disease and most of these patients eventually die from PCa despite significant advances in management $[2 \bullet \bullet]$.

PSA screening was approved by the U.S. Food and Drug Administration (FDA) in 1994. It was designed to reduce the stage at diagnosis, thereby reducing PCa mortality. Screening is typically performed with a digital rectal examination and the prostate specific antigen (PSA) blood test. However, there is a lack of clear consensus regarding many aspects of screening including the frequency of testing, and the age screening should start and end. Several randomized controlled trials of PSA screening have been performed with different protocols and results. PSA screening was associated with a relative PCa mortality reduction of $21 \%$ in the updated European Randomized Study of Screening for Prostate Cancer (ERSPC) [3], but there was no significant reduction in $\mathrm{PCa}$ mortality with screening in the Prostate, Lung, Colorectal and Ovarian Cancer Screening trial (PLCO) [4]. However, both the ERSPC and the PLCO excluded men $\geq 75$ years, presumably assuming that elderly men may not benefit from early detection. As such, the benefits and harms in men $\geq 75$ years are not elucidated by these studies.

According to the U.S. Census, $11.3 \%$ of the U.S. population in 2011 were men $>65$ years [5]. Because of an aging population this demographic will further increase, highlighting the importance of policies to address screening and treatment in this group. Indeed, there is evidence that elderly 
men have higher risk disease and higher $\mathrm{PCa}$ death rates. On the other hand, by age 80 years more than half of American men report at least two prevalent chronic diseases [5]. Because of these competing risks, it is important to distinguish which older men would potentially benefit from screening and treatment, and who will not [6]. This review will highlight observations of the pros and cons of screening and treatment in elderly men from the recent literature.

\section{Screening}

PSA screening gained popularity starting in the early 1990s, and since that time there is an ongoing debate about its utility. A study by Welch estimated that approximately 1 million extra U.S. men have been treated for PCa compared with the time before PSA was introduced [7]. The PCa screening era was heralded by a 1991 study of Catalona et al. [8], in which 1653 healthy men age 50 years and older underwent a serum PSA test. Rectal examination followed by prostatic ultrasonography was performed for PSA values $\geq 4.0 \mathrm{ng} / \mathrm{dl}$. Notably, this study showed that $32 \%$ and $43 \%$ of the cancers would have been missed if rectal examination or ultrasonography alone had been used for screening, respectively. Since that time, the combination of rectal examination and serum PSA measurement has provided a better method of detecting prostate cancer. Interestingly, this early study already pointed out that the value of screening was controversial, especially in the detection of latent prostatic cancers which cause neither morbidity nor mortality.

More than 30 years later, there is still a debate about population-based screening. Although opportunistic screening is common in many countries, most health care systems in Europe have not adopted a population-based screening policy and screening guidelines differ between many professional organizations in the U.S. Survival benefit, quality of life and cost are all important considerations in determining the value of screening.

Several studies have been performed to determine the validity of mass screening for $\mathrm{PCa}[3,4]$. The largest randomized studies on screening are the ERSPC followed by the PLCO trial. In 2012 both studies published an update of their results after 11 and 13 years, respectively. The PCLO found no prostate cancer survival benefit in the screening arm versus the usual care arm (which mostly involved screening); indeed, they describe evidence of harm, especially in the older men. For instance, overdiagnosis and resultant overtreatment might occur regularly in this group. Also, one PLCO analysis reported that comorbidity was associated with the efficacy of screening [9]. The ERSPC update showed that there was a relative risk reduction of $21 \%$ in PCa mortality with screening. During the 10th and 11th year of follow-up, the mortality benefit increased to $38 \%$.
In 2008, the USPSTF recommended against PSA in men aged 75 years and older, and their update in 2012 recommended against PSA based screening for $\mathrm{PCa}$ in all asymptomatic men [10]. Numerous groups have expressed concern about making policy based on only a few randomized trials, rather than population-based data [11]. These trials only test a few specific protocols in a very specific population who are followed for a limited period of time [12]. In particular, screening has greatly evolved since the trials were started, including a much more risk-adapted approach.

\section{Whom Do We Screen?}

The role of screening in the general population has been debated extensively as mentioned in the previous paragraphs. The age to start offering PSA along with the frequency of screening also varies widely. The American Urology Association (AUA) and the American Cancer Society (ACS) advise offering screening for men with a life expectancy of at least 10 years [13]. Traditionally, PCa screening was first discussed at age 50 years, except in men with specific risk factors such as a positive family history. However, both the AUA and NCCN [14•] now recommended checking a PSA level at age 40 years for risk stratification.

Another unresolved issue is when to discontinue screening. In the ERSPC trial, including men ages $<75$ years, screening benefit was restricted to men age 55 to 69 years, although it was not powered for this subset analysis [3]. Some groups have suggested using the absolute PSA level or PSA velocity to help assess the need for further screening in older men $[15,16]$.

Overall, the available data to guide evidence-based decisions in the elderly men are limited. Drazer et al. [17] extracted data from the population-based National Health Interview Survey (NHIS) on rates and predictors of PSA screening among older men in the United States. A total of 2623 men without a history of prostate cancer were included in the analysis. Demographic, socioeconomic, and functional characteristics were collected from 2000 and 2005, and 5-year life expectancy was estimated. The overall PSA screening rate was $23.7 \%$ in 2000 and $26.0 \%$ in 2005 , and varied significantly by age $(\mathrm{P}<0.001)$. In the youngest men ages $40-44$ years, $7.5 \%$ got PSA screening. In men ages $45-49,50-54$, and 55 59 years, the frequency was $11.3 \%, 24.0 \%$, and $31.5 \%$, respectively. The rate peaked to $45.5 \%$ in the $70-74$ years age group, but decreased to $24.6 \%$ in the $\geq 85$ age group. Almost half of men $(47.3 \%)$ age $\geq 70$ years with a good life expectancy ( $\leq 15 \%$ probability of 5 -year mortality) were screened, compared to $30.3 \%$ of men with a poor life expectancy ( $\geq 48 \%$ probability of 5-year mortality). Multivariate analysis for predictors of PSA screening showed higher rates in younger men, 
those with fewer comorbidities, more education, married status, positive family history, saw a GP in the past year, had Medicare coverage, received colorectal cancer screening, performed moderate activity, currently drink alcohol, and use vitamins.

Another study including 597,642 U.S. male veterans ages $\geq 70$ years reported that $56 \%$ received a PSA test in 2003. Among men $\geq 85$ years, a PSA test was performed in $36 \%$ of those with worst health $(<10 \%$ probability of living of ten years) compared to $34 \%$ in best health [18 $]$.

Both studies recommend considering life expectancy in the decision about PSA screening, since excessive screening for PCa in men with a limited life expectancy can result in unnecessary anxiety and complications.

\section{Screening and the Elderly}

When we look in more detail at PSA testing in older men, the USPSTF specifically recommended against PSA screening after age 75 years in 2008. However, a large proportion (78\%) of patients disagreed with this recommendation [19], and it ultimately did not lead to changes in clinical practice as investigated by Scosyrev et al. [20]. They specifically examined the frequency of PSA testing in men aged $\geq 75$ years in the Behavioral Risk Factor Surveillance System (BRFSS) from $2006(\mathrm{n}=9033), 2008(\mathrm{n}=12063)$ and $2010(\mathrm{n}=14782)$. In each year, the data were stratified according to age (76-80 versus $\geq 81$ years) and presence of cardiovascular disease (CVD). The percentages of PSA testing varied between $63 \%$ (76- 80 years) and $56 \%$ ( $\geq 81$ years) in $2006,67 \%$ (7680 years) and $58 \%$ ( $\geq 81$ years) in 2008, and $66 \%$ (7680 years) and $54 \%$ ( $\geq 81$ years) in 2010. CVD status was not significantly associated with the frequency of screening. Thus, there was no substantial reduction in the frequency of PSA testing in elderly men compared with the years before the USPSTF recommendation. A key limitation of this study is that the BRFSS survey did not make a distinction regarding the reason for PSA testing, and whether it was done for screening versus follow-up. Another limitation is that these data were based on self-report so there is a possibility of recall bias.

Similar to the Scosyrev study [20], an older study by Li et al. [21•] described the prevalence and predictors of PSA testing in men aged $\geq 75$ years using data from the BRFSS in 2006. Overall, $60 \%$ of men $\geq 76$ years underwent a PSA test in the past year. Predictors of not receiving PSA screening included age $\geq 81$ years, less than a high school diploma, divorced/widowed/separated, not having insurance, dissatisfaction with life, and less emotional support.

In 2010, Hoffman et al. [22] reported a study of PSA screening in 718 men age 75 years or older based on selfreported health status and life expectancy in the National Health Interview Survey (NHIS). Age was categorized as
$75-79,80-84$, and $\geq 85$ years. Of men ages $75-79$ years, $58.6 \%$ were screened compared to $51.9 \%$ of men ages $80-$ 84 , and $34 \%$ of men aged $\geq 85$ years. After adjusting for characteristics such as age, education, and visit to a doctor in last year and race, there was a significant association between self-reported health status and recent PSA screening: $60.4 \%, 50.1 \%$, and $42.2 \%$ for excellent, good, fair or poor health, respectively. Since it takes at least 10 years to realize a survival benefit from PCa treatment, life expectancy should be an important consideration in the decision. What makes the debate more complicated is the fact that prostate cancer in elderly can be more aggressive.

\section{Do Elderly Men Benefit from Early Detection of Prostate Cancer?}

According to the database of the U.S. National Cancer Institute, the median age at diagnosis of prostate cancer is 68 years [23]. The management of prostate cancer in the elderly had been debated for many years, since it is well known that some cancers will never progress or cause death without treatment. However, in patients with advanced disease, $\mathrm{PCa}$ is usually symptomatic and most of these patients will ultimately die of their disease. To determine the frequency of metastatic prostate cancer in different age groups, records from 464,918 patients who were diagnosed with $\mathrm{PCa}$ were obtained from the Surveillance, Epidemiology, and End Results (SEER) database [2]. Patients were categorized into age groups ranging from $<50$ to $\geq 90$ years. The frequency of metastatic PCa differed from $3 \%$ (age $<75$ years) to $17 \%$ (age $\geq 90$ years). The 5 -year cumulative incidence of death from PCa ranged from $3-4 \%$ (any age $<75$ years) up to $30 \%$ for patients aged $\geq 90$ years. The contribution of men aged $>75$ years to $\mathrm{M} 1$ cases was $52 \%$, while men aged $>75$ years contributed $47 \%$ of PCa deaths. In summary, for older patients the risk of death from PCa was increased. This may be explained by more aggressive disease, fewer invasive diagnostic procedures (such as a biopsy) and/or less aggressive treatment. Physicians may be less willing to perform a biopsy in elderly men. Indeed, both biopsy and treatment may have greater risks in older patients with comorbidities.

Numerous studies have similarly reported more aggressive disease in men ages 70 years or older. Brasell et al. examined [24] 12,081 men from the Prostate Disease Research database, diagnosed with PCa between 1989 and 2009, who were stratified further by age and race. Of all patients aged $\geq 70$ years, $49.4 \%$ had external beam radiation therapy, $24.6 \%$ had radical prostatectomy, $18.7 \%$ had hormonal therapy, $6 \%$ had brachytherapy, and $1.2 \%$ had cryotherapy. Men aged $\geq 70$ years had a significantly higher clinical stage, biopsy grade and prediagnosis PSA velocity $(\mathrm{p}<0.0001)$. Among patients who underwent prostatectomy, 
pathological stage, grade and positive surgical margin rates were also higher in elderly men. Upgrading was also more common in the older group, with $18.8 \%$ upgraded to a Gleason $8-10$ on pathology $(\mathrm{p}=0.015)$. Biochemical recurrence and secondary treatment were significantly more likely in elderly men. Kaplan-Meier overall survival analysis showed improved survival for men aged $\geq 70$ years who received radical prostatectomy or external beam radiation therapy compared with expectant management.

\section{Comorbidities and Life Expectancy}

In general, older people have more comorbidities. A precise estimation of life expectancy is important in the decision whom to screen, whom to treat, and when choosing between different options. Patients with a long life expectancy may be offered aggressive treatment, whereas those with short life expectancy because of underlying disease may be advised to follow a conservative approach. Health status can be assessed by many different indices, for example the WHO status, the Charlson comorbidity index, and the Total Illness Burden Index for prostate cancer. To assess competing risks, Daskivich et al. [25] used Charlson scores in a retrospective study of 1482 men diagnosed with nonmetastatic prostate cancer from 1997 to 2004 . The study had a mean follow-up of 6 years. Older men and those managed by primary androgen deprivation therapy had higher Charlson scores, which in turn were associated with greater non-PCa mortality. Ten years after treatment, men with Charlson scores of 0 had a non-PCa mortality rate of $17 \%$, while men with Charlson scores of $3+$ had a non-PCa mortality rate of $74 \%$. During the observation period, $32 \%$ of men died, of which $3 \%$ died from PCa and $25 \%$ died from other causes. Prostate cancer mortality was extremely rare in the low-risk and intermediate-risk groups, independent of the treatment received. Tewari et al. [26] also calculated a probability of 10 -year overall survival in men with and without prostate cancer using Charlson scores.

The Total Illness Burden Index (TIBI), a patient-reported measure of comorbidity, has also been adapted for use in decision-making among men with PCa. An observational study was performed in 2894 men with PCa from the Cancer of the Prostate Strategic Urologic Research Endeavor (CAPSURE) registry [27]. The goal of this study was to examine how TIBI predicted mortality during the next 3.5 years. Men with the highest TIBI score were 13 times more likely to die of other causes than prostate cancer (HR $13.1,95 \% \mathrm{CI}, 6.3-27.4)$. The 3.5 year overall survival rate was $95.1 \%$ in men with TIBI $1-2$ and $56.1 \%$ in men TIBI $\geq 12$, respectively. These results suggest that several different scales exist which can help assess competing risks among men considering screening or treatment for PCa.

\section{Treatment}

Whom Should We Treat and How Can We Avoid Overtreatment?

If screening is potentially useful for some elderly, the next issue is to understand the advantages and disadvantages of various treatment options for prostate cancer in this population. As with the decision about screening, in any age group, it is important that physicians take clinical condition into consideration when selecting a management strategy. Factors associated with initial treatment selection were explored in a cohort of 276 men with localized PCa diagnosed in $1995-1996$ [28]. A Markov model was used to compare the treatment that was received with the optimal treatment. Treatment was considered "suboptimal" if the difference was 0.2 to 1.0 life years or quality-adjusted life years. Independent predictors of suboptimal treatment were age, Gleason score and comorbidity. Healthy men aged 70 years or older had suboptimal treatment in $46.9 \%$ (Gleason 5 - 7) and $72.7 \%$ (Gleason $8-10$ ) of cases. Most men in this group received watchful waiting when curative therapy was preferred. This study shows that age seems to be a barrier in treatment especially in the case of otherwise healthy older men.

According to the working group of the International Society of Geriatric Oncology, older men with PCa should be managed according to their individual health status. The working group classified patients into four groups for decision making: 1. "Healthy" patients (controlled comorbidity, fully independent in daily living activities, no malnutrition) should receive the same treatment as younger patients, 2 . "Vulnerable" patients (reversible impairment) should receive standard treatment after medical intervention, 3. "Frail" patients (irreversible impairment) should receive adapted treatment, and 4. Patients who are "too sick" with "terminal illness" should receive only symptomatic palliative treatment. An extended summary of the management of prostate cancer in older men by Droz et al. provides all of the recommendations of this working group with practical guidelines $[29 \bullet \bullet]$.

Predictive tools may also be useful in choosing the optimal strategy for the elderly. Different studies have developed useful nomograms to weigh an individual's risk of disease progression against risk of non-cancer death. A comprehensive nomogram was designed by Kutikov et al. [30] in 6091 patients with clinically-localized PCa managed with radical prostatectomy $(n=4117)$ or radiation therapy $(n=1974)$ from the CaPSURE database. Competing risk proportional hazards regression models (Fine and Gray) were used to calculate the risks of $\mathrm{PCa}$-specific and non-PCa death. Median age at diagnosis was 65 years. Patients treated with radiotherapy were older, had a greater number of comorbidities and had more adverse pathological tumor features compared to 
patients who had a radical prostatectomy. During follow-up, 983 men died, including 167 of PCa. Although comorbidities were significantly associated with non-PCa death $(\mathrm{P}<0.01)$, they were not significantly associated with $\mathrm{PCa}$ death $(p=0.44)$. Nomograms were ultimately created that can be used as a clinical tool for patients with localized prostate cancer to quantify the 10-year risk of $\mathrm{PCa}$ versus non-PCa mortality using a combination of age, race, comorbidity status, $\mathrm{PCa}$ risk classification using the Cancer of the Prostate Risk Assessment (CAPRA) score, primary treatment modality, and receipt of androgen deprivation therapy.

\section{How Do We Treat?}

Even after taking age and comorbidities into consideration, the optimal treatment for elderly men with prostate cancer is controversial, particularly after the publication of several randomized trials comparing the long-term results of radical prostatectomy versus watchful waiting (WW). The Scandinavian Prostate Cancer Group Study Number 4 (SPCG-4) showed an overall survival benefit with prostatectomy for men aged $<65$ years [31]. However, subgroup analysis among men $\geq 65$ years showed no reduction in metastases, death from any cause, or death from $\mathrm{PCa}$ with radical prostatectomy over watchful waiting. The groups were small (347 men in radical prostatectomy and 348 men in the watchful waiting group) and consisted mostly of men who were clinically diagnosed with prostate cancer in $1989-1999$. Because the median age of prostate cancer diagnosis is 67 years, it is debatable what is the balance of benefits and risks of surgery in the elderly. More recently, the Prostate Cancer Intervention versus Observation Trial (PIVOT) also assigned men with localized $\mathrm{PCa}$ to prostatectomy or observation, with a mean age of 67 years. The results of this study showed no significant difference in all cause mortality or prostate cancer-specific mortality comparing prostatectomy with observation [32].

Rice et al. [33] studied PCa treatment in 770 men aged 70 years or older with low risk PCa (clinical stage T1 $-2 \mathrm{a}$, Gleason score $\leq 6$, PSA $<10 \mathrm{ng} / \mathrm{ml}$ ) in 1989 to 2009 from the Center for Prostate Disease Research database. Overall, $25 \%$ underwent prostatectomy, $33 \%$ had external beam radiation therapy, and $42 \%$ had initial observation (of whom $34 \%$ eventually received secondary therapy). There were significant differences in age and PSA at diagnosis between men treated by radical prostatectomy, external beam radiotherapy, WW with secondary treatment, and WW without secondary treatment. Specifically, RP patients were younger, while WW patients had the lowest PSA at diagnosis. There were no significant differences in race, comorbidities, and clinical stage between these groups. In the Cox proportional hazards models, radical prostatectomy compared to secondary definitive treatment after a period of observation demonstrated a trend toward higher biochemical recurrence-free survival $(p=0.05)$. Watchful waiting without secondary treatment had significantly lower biochemical recurrence-free and overall survival compared to radical prostatectomy. There were no significant differences in progression-free or overall survival between RP and EBRT after multivariable adjustment. Although this study did not look in detail at high grade disease, it is interesting to note that older men, especially men aged 70 years, were treated less aggressively than younger men independent of comorbidities. The authors conclude that initial conservative management is possible for D'Amico low-risk patients $\geq 75$ years but that definitive treatment should be considered in appropriate candidates "throughout the continuum of care."

Role of Androgen Deprivation Therapy as a Treatment for Elderly Men with Localized Prostate Cancer and Comorbidities

For patients with metastatic $\mathrm{PCa}$, hormonal therapy is the mainstay of treatment. It has also been used to shrink large prostate glands before brachytherapy via the induction of apoptosis in susceptible cells. For men undergoing external beam radiation therapy, concurrent use of ADT has shown improve disease specific survival for higher risk disease [34].

However, the use of PADT for localized disease or NADT before radical prostatectomy is not supported by the evidence. Cooperberg et al. [35] analyzed national trends and predictors of inappropriate PADT and NADT in 3439 patients from the CaPSURE observational database between 1989 and 2001. Patients were classified into low-, intermediate-, and high-risk groups. Time trends showed an increase of PADT from $4.6 \%$ to $14.2 \%$ in low-risk groups, $8.9 \%$ to $19.7 \%$ in intermediate-risk groups and $32.8 \%$ to $48.2 \%$ in high-risk groups $(\mathrm{p}<0.001)$. NADT before radical prostatectomy also increased (from $2.9 \%$ to $7.8 \%, p=0.003$ ). A subset analysis by age showed a significant difference in the use of PADT and NADT between men $<60$ years $(11.8 \%$ and $8.3 \%$ ) and men $\geq 80$ years ( $57.7 \%$ and $22.9 \%$ ), respectively. It is important to ensure that older men are being treated in an evidence-based fashion.

\section{Conclusion}

This review focuses on controversies in PCa screening and treatment in elderly men, which require special consideration. PSA screening is highly prevalent in the U.S., including in elderly men, despite recommendations to the contrary from the USPSTF. Screening and treatment of elderly men with limited life expectancies may contribute to the current problems with overdiagnosis and overtreatment. However, elderly men also have a greater risk of aggressive prostate cancer, and are more likely to develop metastases or die from 
their disease. Although the results of a large study of prostatectomy versus watchful waiting showed no reduction in mortality in subgroup analysis among men $\geq 65$ years, other studies show substantial benefits of early definitive treatment for healthy elderly men with aggressive disease. This highlights a potential advantage of active surveillance compared to watchful waiting, to allow continued monitoring for disease progression in time to offer treatment when necessary. In addition, evidence-based management should still be used for the elderly population, including avoidance of PADT and NADT before radical prostatectomy. Overall, defining the optimal protocol for screening and treatment of prostate cancer is a challenge in every age group, but in elderly men there is significant room for improvement. Screening is not indicated for elderly men with a limited life expectancy who would not be candidates for definitive therapy; whereas, healthy older men with high grade disease should be treated aggressively to avoid undue mortality after a thorough discussion about expectations and complications.

Conflict of Interest Annelies Vellekoop declares she has no conflict of interest.

Stacy Loeb declares she has no conflict of interest.

\section{References}

Papers of particular interest, published recently, have been highlighted as:

- Of importance

.- Of major importance

1. http://www.cancer.org/cancer/prostatecancer/detailedguide/ prostate-cancer-key-statistics. Accessed on February 20, 2013.

2. •- Scosyrev E, Messing EM, Mohile S, Golijanin D, Wu G. Prostate cancer in the elderly: frequency of advanced disease at presentation and disease-specific mortality. Cancer. 2012;118(12):3062-70. doi:10.1002/ cncr.26392. Provides important data about life-threatening prostate cancer in the elderly.

3. Schröder FH, Hugosson J, Roobol MJ, Tammela TL, Ciatto S, Nelen V, et al. Prostate-cancer mortality at 11 years of follow-up. N Engl J Med. 2012;366(11):981-90. doi:10.1056/NEJMoa1113135.

4. Andriole GL, Crawford ED, Grubb 3rd RL, et al. Prostate cancer screening in the randomized Prostate, Lung, Colorectal, and Ovarian Cancer Screening Trial: mortality results after 13 years of follow-up. J Natl Cancer Inst. 2012;104(2):125-32. doi:10.1093/jnci/djr500.

5. United States Census Bureau: Age and Sex 2011. Available at http://www.census.gov/population/age/data/2011.html. Accessed February 20, 2013.

6. Albertsen PC, Fryback DG, Storer BE, Kolon TF, Fine J. Longterm survival among men with conservatively treated localized prostate cancer. JAMA. 1995;274(8):626-31.

7. Welch HG, Albertsen PC. Prostate cancer diagnosis and treatment after the introduction of prostate-specific antigen screening: 19862005. J Natl Cancer Inst. 2009;101(19):1325-9. doi:10.1093/jnci/ djp278.
8. Catalona WJ, Smith DS, Ratliff TL, et al. Measurement of prostate-specific antigen in serum as a screening test for prostate cancer. N Engl J Med. 1991;324(17):1156-61. doi:10.1056/ NEJM199104253241702.

9. Crawford ED, Grubb R 3rd, Black A, Andriole GL Jr, Chen MH, Izmirlian $\mathrm{G}$, et al. Comorbidity and mortality results from a randomized prostate cancer screening trial. J Clin Oncol. 2011;29(4): 355-61. doi:10.1200/JCO.2010.30.5979.

10. U.S. Preventive Services Task Force: Screening for Prostate Cancer Recommendation Statement. May 2012. Available at http:// www.uspreventiveservicestaskforce.org/prostatecancerscreening/ prostatefinalrs.htm. Accessed February 20, 2013.

11. Catalona WJ, D'Amico AV, Fitzgibbons WF, et al. What the U.S. Preventive Services Task Force missed in its prostate cancer screening recommendation. Ann Intern Med. 2012;157(2):137-8. doi:10.7326/0003-4819-157-2-201207170-00463.

12. Etzioni R, Gulati R, Cooperberg MR, Penson DM, Weiss NS, Thompson IM. Limitations of basing screening policies on screening trials: The US Preventive Services Task Force and Prostate Cancer Screening. Medical Care. 2013. doi:10.1097/MLR.0b013e31827da979.

13. American Cancer Society. Available at http://www.cancer.org/ cancer/prostatecancer/moreinformation/prostatecancerearly detection/prostate-cancer-early-detection-acs-recommendations. Accessed March 1, 2013.

14. - National Comprehensive Cancer Network, Treatment Guidelines, Prostate Cancer. Available at http://www.nccn.org/professionals/ physician_gls/pdf/prostate.pdf. National Comprehensive Cancer Network recommendations for prostate cancer treatment.

15. Schaeffer EM, Carter HB, Kettermann A, Loeb S, Ferrucci L, Landis $\mathrm{P}$, et al. Prostate specific antigen testing among the elderly-when to stop? J Urol. 2009;181(4):1606-14; discussion 16134. doi:10.1016/j.juro.2008.11.117.

16. Tang P, Sun L, Uhlman MA, et al. Prostate-specific antigen-based risk-adapted discontinuation of prostate cancer screening in elderly African American and Caucasian American men. Urology. 2010;76(5):1058-62. doi:10.1016/j.urology.2009.09.049.

17. Drazer MW, Huo D, Schonberg MA, Razmaria A, Eggener SE. Population-based patterns and predictors of prostate-specific antigen screening among older men in the United States. Journal of Clinical Oncology. 2011;29(13):1736-43. doi:10.1200/ JCO.2010.31.9004.

18. • Walter LC, Bertenthal D, Lindquist K, Konety BR. PSA screening among elderly men with limited life expectancies. JAMA: the journal of the American Medical Association. 2006;296(19):2336-42. doi:10.1001/jama.296.19.2336. Study of screening patterns in elderly men.

19. Caire AA, Sun L, Robertson CN, et al. Public survey and survival data do not support recommendations to discontinue prostate-specific antigen screening in men at age 75 . Urology. 2010;75(5):1122-7. doi:10.1016/j.urology.2009.06.091.

20. Scosyrev E, Wu G, Golijanin D, Messing E. Prostate-specific antigen testing in older men in the USA: data from the behavioral risk factor surveillance system. BJU Int. 2012;110(10):1485-90. doi:10.1111/ j.1464-410X.2012.11013.x.

21. • Li J, Zhao G, Pollack LA, Smith JL, Joseph DA. Use of the prostatespecific antigen test among men aged 75 years or older in the United States: 2006 Behavioral Risk Factor Surveillance System. Prev Chron Dis. 2010;7(4):A84. PSA testing in elderly men.

22. Hoffman KE, Nguyen PL, Ng AK, D'Amico AV. Prostate cancer screening in men 75 years old or older: an assessment of self-reported health status and life expectancy. J Urol. 2010;183(5):1798-802. doi:10.1016/j.juro.2010.01.002.

23. SEER Stat Fact Sheets: prostate. Secondary SEER Stat Fact Sheets: prostate.

24. Brassell SA, Rice KR, Parker PM, et al. Prostate cancer in men 70 years old or older, indolent or aggressive: clinicopathological 
analysis and outcomes. J Urol. 2011;185(1):132-7. doi:10.1016/ j.juro.2010.09.014.

25. Daskivich TJ, Chamie K, Kwan L, et al. Comorbidity and competing risks for mortality in men with prostate cancer. Cancer. 2011;117(20):4642-50. doi:10.1002/cncr.26104.

26. Tewari A, Johnson CC, Divine G, et al. Long-term survival probability in men with clinically localized prostate cancer: a casecontrol, propensity modeling study stratified by race, age, treatment and comorbidities. J Urol. 2004;171(4):1513-9. doi:10.1097/ 01.ju.0000117975.40782.95.

27. Litwin MS, Greenfield S, Elkin EP, Lubeck DP, Broering JM, Kaplan SH. Assessment of prognosis with the total illness burden index for prostate cancer: aiding clinicians in treatment choice. Cancer. 2007;109(9):1777-83. doi:10.1002/cncr.22615.

28. Schwartz KL, Alibhai SM, Tomlinson G, Naglie G, Krahn MD. Continued undertreatment of older men with localized prostate cancer. Urology. 2003;62(5):860-5.

29. •- Droz JP, Balducci L, Bolla M, et al. Management of prostate cancer in older men: recommendations of a working group of the International Society of Geriatric Oncology. BJU Int. 2010;106(4):462-9. doi:10.1111/j.1464-410X.2010.09334.x. Recommendations on management of elderly men.
30. Kutikov A, Cooperberg MR, Paciorek AT, Uzzo RG, Carroll PR, Boorjian SA. Evaluating prostate cancer mortality and competing risks of death in patients with localized prostate cancer using a comprehensive nomogram. Prostate Cancer Prostatic Dis. 2012;15(4):374-9. doi:10.1038/pcan.2012.21.

31. Bill-Axelson A, Holmberg L, Ruutu M, et al. Radical prostatectomy versus watchful waiting in early prostate cancer. $N$ Engl J Med. 2011;364(18):1708-17. doi:10.1056/NEJMoa1011967.

32. Wilt TJ, Brawer MK, Jones KM, et al. Radical prostatectomy versus observation for localized prostate cancer. N Engl J Med. 2012;367(3):203-13. doi:10.1056/NEJMoa1113162.

33. Rice KR, Colombo ML, Wingate J, et al. Low risk prostate cancer in men $>/=70$ years old: to treat or not to treat. Urol Oncol. 2011. doi:10.1016/j.urolonc.2011.07.004.

34. Bolla M, Van Tienhoven G, Warde P, et al. External irradiation with or without long-term androgen suppression for prostate cancer with high metastatic risk: 10-year results of an EORTC randomised study. Lancet Oncol. 2010;11(11):106673. doi:10.1016/S1470-2045(10)70223-0.

35. Cooperberg MR, Grossfeld GD, Lubeck DP, Carroll PR. National practice patterns and time trends in androgen ablation for localized prostate cancer. J Natl Cancer Inst. 2003;95(13):981-9. 